\title{
Conexão com a natureza e associação com motivos de escolhas alimentares entre profissionais da atenção primária à saúde
}

\author{
Connectedness to nature and its association with food choice \\ motives among primary health care professionals
}

\begin{abstract}
Vânia Hercília Talarico Bruno (https://orcid.org/0000-0002-5101-5435) ${ }^{1}$
Ivan da Silva Beteto (https://orcid.org/0000-0003-2805-0192) ${ }^{1}$

Pedro Henrique Leonetti Habimorad (https://orcid.org/0000-0001-9276-6019) ${ }^{1}$

Fernanda Martin Catarucci (https://orcid.org/0000-0003-2935-2475) ${ }^{1}$

Hélio Rubens Carvalho Nunes (https://orcid.org/0000-0002-7806-1386) ${ }^{1}$

Maria Antonieta de Barros Leite Carvalhães (https://orcid.org/0000-0002-6695-0792) ${ }^{1}$

Karina Pavão Patricio (https://orcid.org/0000-0003-2112-5956) ${ }^{1}$
\end{abstract}

${ }^{1}$ Faculdade de Medicina de Botucatu, Universidade Estadual Paulista Júlio de Mesquita Filho. Av. Bento Lopes 679, Rubião Júnior. 18618-970 Botucatu SP Brasil.

vaniahtalarico@gmail.com

\begin{abstract}
Connectedness to nature can boost well-being and lead to healthier and more sustainable food choices. Health professionals have the potential to be key agents in promoting environmental health. A cross-sectional study was conducted with 146 primary healthcare professionals to determine the association betweennature connectedness and food choicemotives considered important for human and environmental health. We used the 14-item Connectedness to $\mathrm{Na}$ ture Scale (CNS) and the Food Choice Questionnaire (FCQ), consisting of 36 items distributed between nine factors, including "health", "natural content", and "ethical concern". The average CNS score was 53.8 ( \pm 9$)$. The highest scoring factors of the FCQ were sensory appeal and price. Ethical concern was ranked last. There was a significant positive association between degree of nature connectedness and scoring for the factors health ( $p=$ 0.031 ), natural content ( $p=0.001$ ), and ethical concern $(p<0.001)$. The results of this unprecedented studyshow that increased connectedness to nature may lead to healthier and more sustainable food choices.
\end{abstract}

Key words Healthy diet, Health personnel, Environmental health, The environment, Primary healthcare
Resumo A conexão com a natureza pode favorecer o bem-estar e a adoção de práticas alimentares sustentáveis. Profissionais de saúde seriam agentes fundamentais nesta interface, promovendo a saúde ambiental. Estudo transversal com 146 profissionais da atenção primária avaliou a associação entre conexão com a natureza e os motivos para escolhas alimentares consideradas importantes à saúde humana e ambiental. Aplicou-se Escala de Conexão com a Natureza (ECN), contendo 14 itens que medem o quanto a pessoa se sente integrada ao meio ambiente, variando de 14 a 70 pontos; e o Questionário sobre Motivos para as Escolhas Alimentares (FCQ), com 36 itens distribuidos em nove fatores, dentre os quais elegeu-se para este estudo: "Saúde", "Conteúdo Natural" e "Preocupação Ética". A pontuação média na ECN foi de 53,8, ( \pm 9). "Apelo Sensorial" e "Preço" foram os fatores mais pontuados; "Preocupação Ética" ocupou a última posição. Houve associação positiva significativa da ECN com a pontuação nos fatores "Saúde" ( $p=0,031)$, "Conteúdo Natural" $(p=0,001)$ e "Preocupação Ética" $(p<0,001)$. Os resultados desta pesquisa inédita permitiram concluir que aumentar conexão com a natureza pode favorecer escolhas alimentares mais saudáveis e sustentáveis.

Palavras-chave Dieta saudável; Pessoal da saúde, Saúde ambiental, Meio ambiente, Atenção primária à saúde 


\section{Introdução}

Compreender como se dá a conexão das pessoas com a natureza e como esta relação pode modificar valores e atitudes e influenciar na saúde do planeta é um tema relevante no atual cenário de crise ambiental ${ }^{1}$, marcada por questões complexas como o aquecimento global, a produção exagerada de resíduos sólidos, os desastres naturais, o aumento na disseminação de doenças veiculadas pela água e em função da proliferação de vetores, as condições de moradia e saneamento básico deficientes e o sofrimento, principalmente, das populações mais vulneráveis² .

As mudanças climáticas representam a maior ameaça global à saúde do século XXI ${ }^{3}$. Deve-se atentar ao fato de que os níveis dos gases promotores do efeito estufa, que influenciam diretamente nas mudanças climáticas, excederam em muito a variação natural nos últimos anos em função, inclusive, da ação do homem em razão de suas atividades em agricultura ${ }^{4}$.

A agricultura praticada em larga escala, marcada pela utilização de transgênicos e dominada pela indústria de agrotóxicos, pesticidas e fertilizantes químicos, que cresceu $93 \%$ no mundo e $190 \%$ no Brasil entre os anos de 2005 e 2015, é acompanhada por um aumento do consumo de alimentos ultraprocessados, cuja produção e comercialização contribuem não apenas para o cenário das doenças crônicas, mas também para as mudanças climáticas devido ao aumento da produção de gases promotores do efeito estufa ${ }^{5-7}$. Além disso, o excesso de embalagens geradas pelo consumo em larga escala destes alimentos é bastante preocupante, uma vez que elas representam uma parte significativa dos resíduos sólidos urbanos (RSU), do qual o plástico contribui com cerca de $20 \%$ do volume, em toneladas ${ }^{8,9}$.

No entanto, a preocupação com as embalagens não parece ser um fator importante quando se avalia os motivos pelos quais as pessoas escolhem seus alimentos, conforme demonstrado na revisão sistemática de Cunha et al. ${ }^{10}$. Nesta revisão, o autor avaliou os principais motivos de escolhas alimentares de diferentes populações com base em estudos que aplicaram o Food Choice Questionnaire (FCQ), de Steptoe et al. ${ }^{11}$, questionário sobre motivos para escolhas alimentares que considera a "Preocupação Ética", ou seja, a preocupação com a ética ambiental, como um dos nove fatores importantes para estas escolhas; neste fator contempla-se uma questão específica sobre a preocupação com as embalagens dos alimentos. De acordo com esta revisão, a "Preo- cupação Ética" está entre os dois fatores menos pontuados pelas populações em geral. No Brasil, durante o processo de tradução e adaptação cultural do FCQ, Heitor et al. ${ }^{12}$ aplicaram-no a 86 estudantes e observaram que duas das três afirmações presentes no item "Preocupação Ética" foram consideradas como "nada importante" por mais da metade da amostra pesquisada.

Existem poucos dados publicados sobre a visão de profissionais da saúde sobre essa temática. O único estudo encontrado neste sentido foi o de Sushma et al. ${ }^{13}$, mostrando que a "Preocupação Ética" foi mais pontuada do que "Familiaridade", "Conveniência" e "Humor" entre os 159 estudantes de odontologia, indianos, investigados no estudo. Comparando com os achados da revisão de Cunha et al. ${ }^{10}$, pode-se supor haver influência da profissão ou do contexto cultural nesta questão.

Em "Subsídios para Construção da Política Nacional de Saúde Ambiental" ${ }^{14}$ o Ministério da Saúde brasileiro deixa clara a importância de se pensar na saúde ambiental como um aspecto fundamental da promoção e da proteção à saúde dos cidadãos, uma vez que o ambiente ecologicamente equilibrado está em consonância, inclusive, com os princípios e as diretrizes do Sistema Único de Saúde (SUS), como integralidade do cuidado, universalidade, equidade e controle social.

É responsabilidade dos profissionais de saúde entender a importância da interdisciplinaridade entre saúde e meio ambiente e assumir uma postura crítica e reflexiva sobre o modo de produção, o consumo capitalista e os problemas ambientais e de saúde enfrentados pela humanidade na atualidade ${ }^{15}$. Os profissionais de saúde devem atuar como intermediadores do conhecimento científico vigente, contribuindo para a adoção de novos hábitos e condutas de saúde por parte da população ${ }^{16}$.

Por outro lado, evidências mostram que a conexão com a natureza, compreendida pela forma como as pessoas identificam-se com o ambiente natural e as relações que elas formam com a natureza, está associada a um comportamento pró -ambiental, refletindo escolhas alimentares mais sustentáveis ${ }^{17,18}$. Esta conexão também está atrelada a aspectos psicossociais, como o bem-estar subjetivo e os valores humanos ${ }^{1,19}$.

Não localizamos estudos sobre a conexão com a natureza e/ou motivos para as escolhas alimentares, tampouco entre profissionais de saúde $^{20}$. O presente artigo apresenta resultados de pesquisa inédita que avaliou esses dois constructos e a associação entre eles em amostra de 
profissionais atuantes na rede de atenção primária à saúde brasileira.

\section{Métodos}

\section{Delineamento e amostra}

Trata-se de um estudo transversal realizado com profissionais de saúde da Atenção Primária à Saúde (APS) atuantes em município do interior do estado de São Paulo, região Sudeste do Brasil. O município possui população estimada em aproximadamente 145 mil habitantes, Índice de Desenvolvimento Humano (IDH) de 0,8; apresenta $95.8 \%$ de domicílios com esgotamento sanitário adequado e $25.8 \%$ de domicílios urbanos em vias públicas com urbanização adequada ${ }^{21}$.

O tamanho da amostra $(n=135)$ foi estimado com base em uma estimativa de correlação extraída da meta-análise de Capaldi et al. ${ }^{22}$, considerando-se poder de teste de 0,8 e erro tipo I de 0,05 . O nível de significância adotado foi de $0,05(\mathrm{p}<0,05)$. Vale ressaltar que, devido à inexistência de estudos sobre a associação entre a conexão com a natureza e escolhas alimentares, foi utilizada como base para o cálculo amostral a correlação entre conexão com a natureza e o bem -estar, objeto de interesse do projeto maior que deu origem a este estudo.

No período da coleta de dados, atuavam na rede de atenção primária à saúde local 362 profissionais de saúde, distribuídos em 22 diferentes locais de trabalho e pertencentes a 13 categorias profissionais (de acordo com relação fornecida pela Secretaria de Saúde do município), sendo: 46 agentes comunitários de saúde, 99 auxiliares e 31 técnicos de enfermagem, 35 enfermeiros, 22 auxiliares de consultório dentário, 25 dentistas, 18 técnicos em farmácia, 6 farmacêuticos, 3 psicólogos, 2 fisioterapeutas, 3 fonoaudiólogos, 4 nutricionistas e 68 médicos.

Após o cálculo do número amostral mínimo necessário $(\mathrm{n}$ mínimo $=135)$, foi realizado sorteio dos profissionais atuantes nas Unidades Básicas de Saúde (UBS) e Estratégias de Saúde da Família (ESF), respeitando-se a proporcionalidade de cada categoria profissional. Posteriormente, decidiu-se por incluir os profissionais atuantes no Núcleo de Apoio à Saúde da Família (NASF), visto que em nosso município estes profissionais pertencem e desempenham as atividades na APS, e não estavam inicialmente contemplados. Como o número de profissionais atuante no NASF não era grande, todos foram convidados a participar da pesquisa e os que aceitaram o convite foram incluídos. Com isto, duas categorias de profissionais foram incluídas - Assistentes Sociais e Educadores Físicos - e algumas categorias apresentaram uma representatividade maior do que a proporção real dos atuantes no município (isto aconteceu em especial com os nutricionistas).

A amostra final foi composta por 146 profissionais.

O único critério de inclusão foi pertencer à equipe de profissionais da saúde da atenção primária do município.

\section{Ética}

Este projeto recebeu parecer favorável no dia 07 de Junho de 2017 pelo Comitê de Ética em Pesquisa. Todos os participantes assinaram o Termo de Consentimento Livre e Esclarecido (TCLE), consentindo em participar da pesquisa.

\section{Coleta de dados}

A coleta de dados ocorreu no mês de julho de 2017. Os instrumentos da pesquisa foram entregues aos profissionais com devidas orientações para o preenchimento e recolhidos após uma semana. Em apenas 04 unidades, em virtude de disponibilidade de tempo, os questionários foram respondidos no momento da visita.

\section{Variáveis e instrumentos de coleta de dados}

\section{Exposição}

Conexão com a natureza é um constructo que avalia o quanto uma pessoa se sente integrada e conectada ao ambiente, a partir de uma perspectiva afetiva e individual. Foi avaliada pela Escala de Conexão com a Natureza (ECN), instrumento traduzido e validado no Brasil por Pessoa et al. ${ }^{19}$. Contém 14 itens, os quais devem ser respondidos em uma escala do tipo likert de cinco pontos, que variam de 1 (discordo totalmente) a 5 (concordo totalmente); desta forma, a pontuação varia de 14 a 70, sendo que quanto maior a pontuação, maior a conexão com a natureza.

O valor calculado para o coeficiente de Conbrach do instrumento validado no Brasil por Pessoa et al. ${ }^{19}$ foi de 0,82 ; o encontrado para a amostra deste estudo foi de 0,77 .

\section{Desfechos}

Foi aplicada a escala Food Choice Questionnaire (FCQ) desenvolvida por Steptoe et al. ${ }^{11}$, com tradução e adaptação cultural no Brasil realizada 
por Heitor et al. ${ }^{12}$. Este instrumento autoaplicável avalia nove fatores ou motivos relacionados às escolhas alimentares: 1) Saúde; 2) Humor; 3 ) Conveniência; 4) Apelo Sensorial; 5) Conteúdo Natural; 6) Preço; 7) Controle de Peso; 8) Familiaridade e 9) Preocupação Ética. Resumidamente, o instrumento convida a pessoa a refletir sobre as declarações como, por exemplo, Para mim é importante que o alimento que eu coma no dia-adia seja..., e indicar, dentro de cada fator, os aspectos que ela considera serem mais importantes para determinar sua escolha alimentar.

Dentre esses nove fatores, escolhemos três como variáveis desfechos nas análises para identificar associação entre conexão com a natureza e motivos para as escolhas alimentares: fator 1 (saúde), com variação de 6 a 25; fator 5 (conteúdo natural), com variação de 3 a 12; fator 9 (preocupação ética), com variação de 3 a 12. Escolhemos esses três fatores específicos (1, 5 e 9) em detrimento dos demais do questionário, com base na literatura que aponta que pessoas mais conectadas à natureza preocupam-se em fazer escolhas mais saudáveis (aspectos que são considerados pelos fatores 1 e 5 do FCQ), e sustentáveis (avaliado, de alguma forma, pelo fator 9 do FCQ $)^{6,23}$.

O coeficiente de Cronbach encontrado com a aplicação do FCQ para a amostra deste estudo foi de 0,92 , enquanto para o questionário original de Steptoe et al. ${ }^{11}$ este valor variou de 0,70 a 0,87 e Heitor et al. ${ }^{12}$ encontraram valores variando entre 0,75 e 0,89 .

\section{Potenciais confundidores}

Os potenciais confundidores escolhidos foram sexo, idade $(<35,35-50,>50$; ref $=<35$ anos); graduação (sim ou não); tempo de serviço na área da saúde $(<5,5-10,>10$; ref $=<5$ anos $)$; saúde autorreferida (bom, muito bom, regular, ruim, muito ruim) e prática de atividade física ( $\operatorname{sim}$ ou não).

Estudos apontam que as mulheres em geral preocupam-se mais com a saúde do que os homens e que há diferenças entre as faixas etárias e o nível sociocultural quando se considera a qualidade da dieta, que, por sua vez, relaciona-se às escolhas alimentares ${ }^{24,25}$. Há evidências, ainda, de que a prática de atividade física possa influenciar a escolha por alimentos mais saudáveis ${ }^{26}$. Tais dados foram obtidos por meio de um questionário sócio demográfico, autoaplicado, juntamente com os questionários ECN e FCQ.

\section{Análises}

Os dados foram tabulados e armazenados em banco de dados EPI-INFO Versão Windows, submetidos às análises de consistência e, em seguida, foram processadas estatísticas descritivas.

A associação entre conexão com a natureza e pontuação nos três motivos para escolhas alimentares (fatores 1, 5 e 9 do FCQ), separadamente, foram investigadas por regressão linear múltipla com resposta normal, incluindo os potenciais confundidores, selecionadas as covariáveis que apresentaram associação com os desfechos em nível de $\mathrm{p}<0,20$ em análises prévias univariadas. As associações de interesse foram consideradas estatisticamente significativas se $\mathrm{p}<0,05$. As análises foram feitas com software SPSS v 21.

\section{Resultados}

Foram estudados 146 profissionais atuantes na APS. A Tabela 1 apresenta o número de profissionais entrevistados por categoria.

A amostra do estudo foi composta em sua maioria por mulheres $(82,3 \%)$, cor branca $(83,6 \%)$, faixa etária entre 20 e 50 anos $(84,9 \%)$, graduados ou pós-graduados $(58,2 \%)$, moradores da zona urbana da cidade $(96,6 \%)$ e pessoas que trabalham na área da saúde há mais de 5 anos $(72,60 \%)$.

A pontuação média obtida na Escala de Conexão com a natureza foi de 53,8, com desvio padrão de 9 pontos. $\mathrm{O}$ valor encontrado para o $1^{\circ}$ tercil foi de 52,0 e para o $2^{\circ}$ foi de 59 . O valor médio por questão foi de 3,84 . A pontuação mínima obtida para esta escala foi de 26 e a máxima de 70 pontos (dados não mostrados em tabela).

As pontuações médias obtidas em cada fator do FCQ encontram-se na Tabela 2. Verificou-se que o fator 4 "Apelo Sensorial" foi o mais pontuado entre os profissionais, com uma média ponderada por afirmação de 3,67 pontos, enquanto o fator "Preocupação Ética" foi menos pontuado, com uma média ponderada por afirmação de 2,60. "Saúde" ficou em terceiro lugar e "Conteúdo Natural” obteve a quinta colocação.

Foram encontrados os seguintes escores para o fator "Saúde" (pontuação mínima de 6 e máxima de 24): mediana $=21$ (sendo $1^{\circ}$ quartil 18 e $3^{\circ}$ quartil 23) e intervalo interquartílico de 5. Para o fator "Conteúdo Natural” (pontuação mínima de 3 e máxima de 12) a mediana foi de 10 (sendo $1^{\circ}$ quartil 8 e $3^{\circ}$ quartil 12) e o intervalo interquartílico foi de 4. Já para o fator "Preocupação 
Ética" (pontuação mínima de 3 e máxima de 12) a mediana foi de 8 (sendo $1^{\circ}$ quartil 6 e $3^{\circ}$ quartil 10), com intervalo interquartílico de 4 (dados não apresentados em tabela).

Observou-se uma associação bruta positiva significativa entre a pontuação na ECN e os fatores "Saúde" ( $\mathrm{p}=0,023)$, "Conteúdo Natural" ( $\mathrm{p}=$ $0,002)$ e "Preocupação Ética" ( $p<0,001)$ (dados não mostrados em tabela).

Conforme apresenta a Tabela 3, pode-se observar que a pontuação na ECN se associa po-

Tabela 1. Distribuição dos profissionais participantes da pesquisa por categoria.

\begin{tabular}{lcr}
\hline Categoria profissional & $\begin{array}{c}\text { Número de } \\
\text { participantes }\end{array}$ & \multicolumn{1}{c}{$\%$} \\
\hline Agente comunitário de & 18 & 12,3 \\
saúde & & \\
Assistente Social & 2 & 1,4 \\
Auxiliar de consultório & 6 & 4,1 \\
dentário & & \\
Auxiliar de enfermagem & 33 & 22,6 \\
Auxiliar de farmácia & 6 & 4,1 \\
Dentista & 9 & 6,2 \\
Educador Físico & 2 & 1,4 \\
Enfermeiro & 16 & 11,0 \\
Farmacêutico & 3 & 2,1 \\
Fonoaudiologista & 1 & 0,7 \\
Fisioterapeuta & 5 & 3,4 \\
Médico & 22 & 15,1 \\
Nutricionista & 7 & 4,8 \\
Psicólogo & 4 & 2,7 \\
Técnico de Enfermagem & 12 & 8,2 \\
Total & 146 & 100,0 \\
\hline
\end{tabular}

sitiva e significantemente ao fator 1 ("Saúde") do FCQ $(\mathrm{p}=0,031)$, em análise ajustada para os confundidores sexo, idade, graduação, tempo de serviço na área da saúde, saúde autorreferida. Ocorre um aumento médio de 0.07 pontos no FCQ1 a cada ponto na conexão com a natureza. Existe também uma associação significativa entre a pontuação na ECN e o fator 5 ("Conteúdo Natural”) do FCQ. Há um aumento médio de 0.06 pontos no FCQ5 a cada ponto na conexão com a natureza $(\mathrm{p}=0,001)$ após ajuste para os confundidores idade e tempo de serviço na área da saúde. Há, ainda, uma associação significativa entre a pontuação na ECN e o fator 9 ("Preocupação Ética") do FCQ, após ajuste para idade, escolaridade e tempo de serviço na área da saúde. Ocorre um aumento médio de 0.09 pontos no FCQ 9 a cada ponto na conexão com a natureza $(\mathrm{p}<0,001)$.

\section{Discussão}

Este estudo propôs-se a avaliar a associação entre intensidade de conexão com a natureza, medida com a Escala de Conexão com a Natureza, e o grau de concordância do indivíduo sobre três motivos para escolhas alimentares considerados importantes do ponto de vista da saúde humana e ambiental: "Saúde", "Conteúdo Natural" e "Preocupação Ética”. Já a escolha por avaliar essa relação em amostra de profissionais de saúde da atenção primária foi baseada no potencial papel que estes profissionais podem assumir como educadores em saúde ${ }^{15,16,27}$. Segundo nosso conhecimento, é o primeiro estudo, em nível nacional e internacional, que investigou esta associação nesse grupo populacional ${ }^{20}$.

Tabela 2. Distribuição da pontuação nos fatores do Questionário sobre Motivos para as Escolhas Alimentares (FCQ) pelos profissionais da saúde da APS ( $\mathrm{n}=146)$.

\begin{tabular}{|c|c|c|c|c|c|c|c|c|c|}
\hline \multirow[b]{3}{*}{ Medida } & \multicolumn{9}{|c|}{ Fatores do FCQ } \\
\hline & F1 & F2 & F3 & F4 & F5 & F6 & F7 & F8 & F9 \\
\hline & Saúde & Humor & Conveniência & $\begin{array}{c}\text { Apelo } \\
\text { Sensorial }\end{array}$ & $\begin{array}{l}\text { Conteúdo } \\
\text { Natural }\end{array}$ & Preço & $\begin{array}{c}\text { Controle } \\
\text { de Peso }\end{array}$ & Familiaridade & $\begin{array}{c}\text { Preocupação } \\
\text { Ética }\end{array}$ \\
\hline Média & 20,08 & 18,75 & 16,59 & 14,70 & 9,75 & 10,07 & 9,25 & 8,39 & 7,82 \\
\hline $\begin{array}{l}\text { por } \\
\text { fator } \\
\pm D P\end{array}$ & $\pm 3,57$ & $\pm 5,01$ & $\pm 3,57$ & $\pm 2,06$ & $\pm 2,34$ & $\pm 1,92$ & $\pm 2,64$ & $\pm 2,63$ & $\pm 2,84$ \\
\hline MP & 3,35 & 3,12 & 3,32 & 3,67 & 3,25 & 3,36 & 3,08 & 2,80 & 2,61 \\
\hline$\pm \mathrm{DP}$ & $\pm 0,59$ & $\pm 0,83$ & $\pm 0,71$ & $\pm 0,51$ & $\pm 0,78$ & $\pm 0,64$ & $\pm 0,88$ & $\pm 0,88$ & $\pm 0,95$ \\
\hline
\end{tabular}

DP: Desvio Padrão; MP: Média Ponderada. 
Tabela 3. Associação entre pontuação na Escala de Conexão com a Natureza (ECN) e fatores "Saúde”, "Conteúdo Natural” e "Preocupação Ética” do Questionário sobre Motivos para as Escolhas Alimentares (FCQ) corrigida para os potenciais confundidores entre profissionais da atenção primaria a saúde $(\mathrm{n}=146)$.

\begin{tabular}{cccc}
\hline & Saúde $^{*}$ & Conteúdo Natural $^{* *}$ & Preocupação Ética $^{* * *}$ \\
\cline { 2 - 4 } & $\beta(\mathrm{IC} 95 \%) ; \mathrm{p}$ & $\beta(\mathrm{IC}$ 95\%); & $\beta(\mathrm{IC} 95 \%) ; \mathrm{p}$ \\
Pontuação da ECN & $0,07(0,01$ a 0,13$) ; \mathbf{0 , 0 3 1}$ & $0,06(0,03$ a 0,10$) ; \mathbf{0 , 0 0 1}$ & $0,09(0,04$ a 0,14$) ;<\mathbf{0 , 0 0 1}$ \\
\hline
\end{tabular}

*Ajustado por sexo, idade, graduação, tempo de serviço na área da saúde, saúde autorreferida. ${ }^{* *}$ Ajustado para idade e tempo de serviço na área da saúde. ${ }^{* *}$ Ajustado para idade, escolaridade e tempo de serviço na área da saúde.

Os resultados indicaram que os profissionais da atenção primária à saúde, entre eles médicos, enfermeiros, técnicos de enfermagem, dentistas, agentes comunitários de saúde, e outros, têm baixa conexão com a natureza e preocupação ética como motivo para nortear suas escolhas alimentares. Ainda assim, detectou-se uma associação positiva entre conexão com a natureza e a pontuação nos itens saúde, conteúdo natural e preocupação ética relacionados aos motivos para as escolhas alimentares. Esse resultado sugere que ampliar a conexão com a natureza possa ser um caminho para aumentar as possibilidades de que tais profissionais assumam papel de destaque em ações de educação ambiental e alimentar junto à comunidade por eles assistidas, como proposto no documento elaborado pelo Ministério da Saúde (MS) com apoio da Organização Pan-americana de Saúde (OPAS), da Associação Brasileira de Saúde Coletiva (Abrasco) e do Conselho Nacional de Saúde (CNS) sobre "Subsídios para Construção da Política Nacional de Saúde Ambiental” ${ }^{4}$.

Para aferir constructos tão complexos como conexão com a natureza e motivos para escolhas alimentares, utilizamos escalas desenvolvidas por pesquisadores reconhecidos ${ }^{11,28}$ e já traduzidas para o português no Brasil ${ }^{12,19}$ de modo que os resultados acima sintetizados podem ser considerados válidos e úteis para ampliar a escassa literatura nacional e internacional sobre esses temas ${ }^{20}$. Como limitação, entretanto, deve-se lembrar que houve diferença na situação de coleta dos dados, pois parte dos profissionais responderam aos questionários na presença do pesquisador, logo após a obtenção do termo de consentimento, enquanto outros, em função da indisponibilidade de tempo, os levaram para casa e os devolveram preenchidos posteriormente; além disso, uma parte da amostra se deu por conveniência. Outro aspecto a considerar, ainda, é o pequeno tamanho da amostra para teste da associação de interesse, ou seja, o erro do tipo II pode estar acima de 0,20; assim, recomendam-se maiores amostras e padronização da situação de coleta em futuros estudos.

A pontuação média por questão $(3,84)$ obtida na Escala de Conexão com a Natureza assemelhase aos valores encontrados por Rosa et al. ${ }^{29} \mathrm{com}$ 224 estudantes brasileiros de diferentes cursos de uma Universidade do Nordeste $(3,83)$ e é um pouco superior a obtida por estudos realizados com 322 estudantes franceses $(3,33)^{30}$ e 324 estudantes canadenses $(3.28)^{31}$. No entanto, é bastante inferior à encontrada em um estudo realizado na Suécia com 1.320 consumidores de um supermercado, que obteve uma pontuação média de 4,92 por questão em uma versão reduzida da $\mathrm{ECN}^{23}$. Apesar da menor comparabilidade, pelo uso de escala menor, os estudos citados apontam para a influência cultural na intensidade de conexão com a natureza.

É necessário destacar que o motivo menos considerado pelos profissionais de saúde foi a "Preocupação Ética". Quando apresentada a questão Para mim é importante que o alimento que eu coma no dia a dia..., houve baixa pontuação nas respostas referentes a este fator, que eram: - "Venha de países que eu aprove a forma como os alimentos são produzidos"; "Mostre com clareza a identificação do país de origem"; "Seja embalado de forma que não prejudique o ambiente", não se diferenciando da população em geral, que ainda não refere intensamente esses aspectos como motivações para suas escolhas alimentares ${ }^{10}$.

Por outro lado, os profissionais avaliados por nós também não atribuem muita importância à questão da presença de aditivos químicos e ingredientes artificiais quando pensam nos motivos pelos quais fazem suas escolhas de alimentos. O fator "Conteúdo Natural", que obteve o quinto lugar em pontuação, traz as seguintes afirmações: "Não contenha aditivos"; "Contenha ingredientes naturais"; "Não contenha ingredientes artificiais".

Os motivos para as escolhas alimentares, da mesma forma que a conexão com a natureza, também parecem sofrer uma influência cultural, 
como sugere a comparação dos nossos resultados com os verificados em amostra de 165 mulheres japonesas ${ }^{32}$, grupo no qual a "Preocupação Ética" foi o $4^{\circ}$ fator mais pontuado e, ainda, com estudos realizados na Itália $(\mathrm{n}=163)^{33}$, Polônia $(\mathrm{n}=$ $1045)^{33}$ e Taiwan $(\mathrm{n}=263)^{32}$, países nos quais as escolhas alimentares foram norteadas considerando como fator mais importante o impacto da presença de aditivos e ingredientes artificiais.

Esses resultados nos rememetem às recomendações do Guia Alimentar para a População Brasileira $^{34}$, que tratam exaustivamente dos riscos do excesso de consumo de aditivos alimentares característicos dos alimentos ultraprocessados. É crescente o número de pesquisas que se propõem a investigar os mafelícios dos aditivos para a saúde e que já apontam evidências da associação entre o consumo abusivo e rinites e alergias (principalmente corantes), influência no comportamento infantil (hiperatividade), inflamação da microbiota e disbiose (emulsificantes e edulcorantes), além de síndrome metabólica ${ }^{35,36}$.

Não encontramos na literatura nenhum estudo que tenha investigado a conexão com a natureza em relação aos motivos para escolhas alimentares. No entanto, um dos estudos conduzidos pela coorte observacional francesa iniciada no ano de 2009, NutriNet-Santé, se propôs a avaliar motivos para escolhas alimentares comparando-os com a qualidade da dieta. Este estudo ( $\mathrm{n}=31.842$ adultos) mostrou que as pessoas que apresentam uma dieta considerada mais saudável são também mais preocupadas com a questão da ética ambiental, com a produção local de alimentos, assim como com a saúde e a garantia de ausência de contaminantes ${ }^{37}$.

Considerando os resultados encontrados em nosso estudo e frente às recomendações do $\mathrm{Mi}$ nistério da Saúde ${ }^{14}$ e do Guia Alimentar para a População Brasileira ${ }^{34}$ cabe-nos questionar se estes profissionais estariam preparados e motivados para atuarem como promotores da saúde e disseminadores da importância de uma alimentação saudável e sustentável junto aos usuários das unidades de saúde onde atuam. É possível que estes profissinais não tenham recebido, durante sua formação, capacitações com enfoque da saúde ou da educação ambiental capazes de estimular novos hábitos e atitudes. É possível, também, que tenham pouco tempo de contato com ambientes naturais como parques, florestas e cachoeiras, contato este que pode ajudar a promover uma maior conexão com a natureza e um maior comportamento pró-ambiental ${ }^{1}$, além de favorecer a saúde física e mental ${ }^{38}$.
Assim, parece importante pensar/repensar a forma como acontece a educação ambiental nos cursos de graduação em saúde brasileiros e os conteúdos abordados em ações de educação continuada. É necessário que haja um olhar mais atencioso para a educação ambiental voltada aos próprios profissionais de saúde, tanto em seu âmbito de atuação, como durante a graduação, principalmente para aqueles que atuam na atenção primária e que têm a responsabilidade de transmitir este conhecimento à população ${ }^{15,27}$.

Discutindo a importância de o setor da saúde assumir um papel protagonista no cenário das mudanças climáticas, McMichael et al. ${ }^{39}$ propõem que os profissionais incentivem a população a repensar suas escolhas alimentares, favorecendo as práticas produtivas que não apresentem riscos à saúde. Walpole et al. ${ }^{40}$ esclarecem a necessidade de haver espaço no currículo médico para as questões ambientais, de forma que os futuros profissionais assumam um papel de protagonista, através do desenvolvimento de atitudes positivas para a sustentabilidade ambiental e de ferramentas que os capacite como educadores nesta temática.

Em síntese, nossos resultados indicam que os profissionais de saúde, no contexto do estudo, não tem condições de atuarem como promotores da saúde ambiental. É necessário que haja uma ampliação do conhecimento em relação ao impacto do meio ambiente como um importante determinante do processo saúde-doença, para que este conhecimento possa ser norteador de escolhas mais saudáveis e sustentáveis para eles e, por conseguinte, para os usuários por eles assistidos.

Acreditamos que ações e projetos que favoreçam um maior contato desses profissionais com a natureza, como caminhadas em parques ou praças arborizadas e trilhas em ambientes mais preservados, seguindo os modelos de terapia de floresta realizados no Japão, podem auxiliá-los na ampliação de sua conexão com a natureza e, consequentemente, em um melhor comportamento pró-ambiental ${ }^{1,38}$.

\section{Conclusão}

Profissionais da atenção primária à saúde têm pouca conexão com a natureza e a preocupação ética ambiental ocupa o último lugar dentre os motivos por eles considerados relevantes para suas escolhas alimentares, pautadas principalmente pelos aspectos sensoriais dos alimentos e 
seu custo. Ainda assim, detectou-se associação positiva entre intensidade da conexão com a natureza e a pontuação nos fatores "Saúde", "Conteúdo Natural” e "Preocupação Ética” como motivos para tais escolhas.

\section{Colaboradores}

VHT Bruno - Responsável pela pesquisa (Projeto de Mestrado). Participou de todas as fases da pesquisa (submissão do projeto ao Comitê de Ética, coleta de dados, tabulação, análise dos resultados) e escrita do manuscrito. IS Beteto Participou do desenho do estudo, coleta de dados e revisão do manuscrito. PHL Habimorad e FM Catarucci - Participou do desenho do estudo, análise dos resultados e revisão do manuscrito. HRC Nunes - Participou do desenho do estudo, responsável pela análise estatística e revisão do manuscrito. KP Patrício - Participou do desenho do estudo, análise dos resultados, revisão do manuscrito e foi a orientadora do projeto. MABL Carvalhães - Participou da análise estatística e da análise dos resultados, participou da escrita original e revisão do manuscrito. 


\section{Referências}

1. Restall B, Conrad E. A literature review of connectedness to nature and its potential for environmental management. J Environ Manage 2015; 15(159):264-278.

2. World Health Organization. Secretarial of the Convention on Biological Diversity. Connecting global priorities: biodiversity and human health: a state of knowledge review [monograph on the Internet]. Geneva:WHO; 2015 [cited 25 Jun 2009]. 364 p. Available from: https://www.cbd.int/health/SOK-biodiversity -en.pdf.

3. Costello A, Abbas M, Allen A, Ball S, Bell S, Bellamy R, Friel S, Groce N, Johnson A, Kett M, Lee M, Levy C, Maslin M, McCoy D, McGuire B, Montgomery H, Napier D, Pagel C, Patel J, de Oliveira JA, Redclift N, Rees H, Rogger D, Scott J, Stephenson J, Twigg J, Wolff J, Patterson C. Managing the health effects of climate change: Lancet and University College London Institute for Global Health Commission. Lancet 2009; 373(9676):1693-1733.

4. The Intergovernmental Panel on Climate Change. IPCC Fourth Assessment Report: climate change 2007 [monograph on the Internet]. Geneva: IPCC; 2007 [cited 25 Jun 2018]. Available from: https://www.ipcc. ch/publications_and_data/ar4/wg1/en/spmsspm-human-and.html

5. Carneiro FF, Silva LGA, Rigotto RM, Friedrich K, Búrigo AC, organizadores. Dossiê Abrasco: um alerta sobre os impactos dos agrotóxicos na saúde. Rio de Janeiro: EPSJV; São Paulo: Expressão Popular; 2015.

6. Ghvanidze S, Velikova N, Dodd TH, Oldewage-Theron W. Consumers' environmental and ethical consciousness and the use of the related food products information: the role of perceived consumer effectiveness. Appetite 2016; 1(107):311-322.

7. Ribeiro H, Jaime $P$, Ventura D. Alimentação e sustentabilidade. Estud Avanç 2017; 31(89):185-198.

8. Associação Brasileira de Empresas de Limpeza Pública e Resíduos Especiais - ABRELPE. Panorama dos resíduos sólidos no Brasil [monografia na Internet]. São Paulo: ABRELPE; 2016 [acessado 2018 Jun 28]. Disponível em: http://www.abrelpe.org.br/Panorama/ panorama2016.pdf

9. Landim APM, Bernardo CO, Martins IBA, Francisco $M R$, Santos MB, Melo NR. Sustentabilidade quanto às embalagens de alimentos no Brasil. Políms: Ciênc Tecnol [periódico na Internet]. 2016; 26:82-92 [acessado 2018 Jun 28]. Disponível em: https://www.redalyc. org/articulo.oa?id $=47043582013$

10. Cunha LM, Cabral D, Moura AP, Almeida MDV. Application of the food choice questionnaire across cultures: Systematic review of cross-cultural and single country studies. Food Qual Prefer. 2018; 64:21-36.

11. Steptoe A, Pollard TM, Wardle J. Development of a measure of the motives underlying the selection of food: the Food Choice Questionnaire. Appetite 1995; 25(3):267-284

12. Heitor SFD, Estima CCP, Neves FJ, Aguiar AS, Castro SS, Ferreira JES. Tradução e adaptação cultural do questionário sobre motivo das escolhas alimentares (Food Choice Questionnaire - FCQ) para a língua portuguesa. Cien Saude Colet 2015; 20(8):2339-2346.
13. Sushma R, Vanamala N, Nagabhushana D, Maurya M, Sunitha S, Reddy C. Food choice motives among the students of a dental institution in Mysore City, India. Ann Med Health Sci Res 2014; 4(5):802-805.

14. Brasil. Ministério da Saúde (MS). Conselho Nacional de Saúde. Subsídios para construção da Política Nacional de Saúde Ambiental. Brasília: Editora do MS; 2009. (Série B. Textos Básicos de Saúde).

15. Santos DAS, Azevedo PV, Azevedo JVV, Alves TLB, Souza AR. Percorrendo os caminhos da relação entre as políticas públicas de saúde e do meio ambiente. Rev Polit Públicas 2016; 20(1):137-152.

16. Alves VS. Um modelo de educação em saúde para o Programa Saúde da Família: pela integralidade da atenção e reorientação do modelo assistencial. Interface (Botucatu) 2005; 9(16):39-52.

17. Dowd K, Burke KJ. The influence of ethical values and food choice motivations on intentions to purchase sustainably sourced foods. Appetite 2013; 69:137-144.

18. Lewis M, Townsend M. 'Ecological embeddedness' and its public health implications: findings from an exploratory study. Ecohealth 2015; 12(2):244-252.

19. Pessoa, VS, Gouveia VV, Soares AKS, Vilar R, Freires LA. Escala de conexão com a natureza: evidências psicométricas no contexto brasileiro. Estud Psicol 2016; 33(2):271-282

20. Uhlmann K, Lin BB, Ro H. Who cares? The importance of emotional connections with nature to ensure food security and wellbeing in cities. Sustainability 2018; 10(6):1-16.

21. Instituto Brasileiro de Geografia e Estatística. Botucatu: Panorama [monografia na Internet]. Brasília: IBGE; 2019 [acessado 2019 Set 19]. Disponível em: https://cidades.ibge.gov.br/brasil/sp/botucatu/panorama

22. Capaldi CA, Dopko RL, Zelenski JM. The relationship between nature connectedness and happiness: a meta -analysis. Front Psychol 2014; 5:976.

23. Spendrup S, Hunter E, Isgren E. Exploring the relationship between nature sounds, connectedness to nature, mood and willingness to buy sustainable food: a retail field experiment. Appetite 2016; 1(100):133-141.

24. McGowan L, Pot GK, Stephen AM, Lavelle F, Spence M, Raats M, Hollywood L, McDowell D, McCloat A, Mooney E, Caraher M, Dean M. The influence of socio-demographic, psychological and knowledge -related variables alongside perceived cooking and food skills abilities in the prediction of diet quality in adults: a nationally representative cross-sectional study. Int J Behav Nutr Phys Act 2016; 13(1):111.

25. Sijtsma FP, Meyer KA, Steffen LM, Shikany JM, Van Horn L, Harnack L, Kromhout D, Jacobs Junior DR. Longitudinal trends in diet and effects of sex, race, and education on dietary quality score change: the coronary artery risk development in young adults study. Am J Clin Nutr 2012; 95(3):580-586.

26. Alexandre VP, Peixoto MRG, Schmitz BAS, Moura EC. Fatores associados às práticas alimentares da população adulta de Goiânia, Goiás, Brasil. Rev Bras Epidemiol 2014; 17(1):267-280. 
27. Brasil. Ministério da Saúde (MS). Fundação Nacional de Saúde. Diretrizes de educação em saúde visando à promoção da saúde: documento base - documento $I$. Brasília: FUNASA; 2007.

28. Mayer FS, Frantz CM. The connectedness to nature scale: A measure of individuals feeling in community with nature. J Environ Psychol 2004; 24(4):503-515.

29. Rosa CD, Profice CC, Collado S. nature experiences and adults' self-reported pro-environmental behaviors: the role of connectedness to nature and childhood nature experiences. Front Psychol 2018; 26(9):1-10.

30. Navarro O, Olivos P, Fleury-Bahi G. "Connectedness to Nature Scale": Validity and reliability in the french context. Front Psychol 2017; 8(2180):1-8.

31. Lee K, Ashton MC, Choi J, Zachariassen K. Connectedness to nature and to humanity: their association and personality correlates. Front Psychol 2015; 6(1003):1-11.

32. Prescott J, Young O, O'neill L, Yau NJN, Stevens R. Motives for food choice: A comparison of consumers from Japan, Taiwan, Malaysia and New Zealand. Food Qual Prefer 2002; 13(7):489-495.

33. Markovina J, Stewart-Knox BJ, Rankin A, Gibney M, de Almeida MDV, Fischer A, Kuznesoff SA, Poínhos R, Panzone L, Frewerf LJ. Food4Me study: Validity and reliability of Food Choice Questionnaire in 9 European countries. Food Qual Prefer 2015; 45:26-32.

34. Brasil. Ministério da Saúde (MS). Secretaria de Atenção à Saúde. Departamento de Atenção Básica. Guia alimentar para a população brasileira. $2^{\mathrm{a}}$ ed. $1^{\mathrm{a}}$ reimpr. Brasília: MS; 2014.

35. Paula Neto HA, Ausina P, Gomez LS, Leandro JGB, Zancan P, Sola-Penna M. Effects of Food additives on immune cells as contributors to body weight gain and immune-mediated metabolic dysregulation. Front Immunol 2017; 8(1478):1-11.
36. Polônio MLT, Peres F. Consumo de aditivos alimentares e efeitos à saúde: desafios para a saúde pública brasileira. Cad Saude Publica 2009; 25(8):1653-1666.

37. Allès B, Péneau S, Kesse-Guyot E, Baudry J, Hercberg S, Méjean C. Food choice motives including sustainability during purchasing are associated with a healthy dietary pattern in French adults. Nutr J 2017; 16(58):1-12.

38. Cox DT, Shanahan DF, Hudson HL, Fuller RA, Anderson K, Hancock S, Gaston KJ. Doses of nearby nature simultaneously associated with multiple health benefits. Int J Environ Res Public Health 2017; 14(172):8-13.

39. McMichael AJ, Neira M, Bertollini R, Campbell-Lendrum D, Hales S. Climate change: a time of need and opportunity for the health sector. Lancet 2009; 374(9707):2123-2125.

40. Walpole SC, Vyas A, Maxwell J, Canny BJ, Woollard R, Wellbery C, Leedham-Green KE Musaeus P, Tufail-Hanif U, Pavão Patrício K, Rother HA. Building an environmentally accountable medical curriculum through international collaboration. Med Teach 2017; 39(10):1040-1050.

Artigo apresentado em 22/10/2018

Aprovado em 23/06/2019

Versão final apresentada em 25/06/2019

Editores-chefes: Romeu Gomes, Antônio Augusto Moura da Silva 